\title{
The study of frequency-scan photothermal reflectance technique for thermal diffusivity measurement
}

Zilong Hua, Heng Ban', and David H. Hurley

Citation: Review of Scientific Instruments 86, 054901 (2015); doi: 10.1063/1.4919609

View online: http://dx.doi.org/10.1063/1.4919609

View Table of Contents: http://aip.scitation.org/toc/rsi/86/5

Published by the American Institute of Physics

\section{Articles you may be interested in}

Local measurement of thermal conductivity and diffusivity

Review of Scientific Instruments 86, 123901 (2015); 10.1063/1.4936213

A frequency-domain thermoreflectance method for the characterization of thermal properties

Review of Scientific Instruments 80, 094901 (2009); 10.1063/1.3212673

Transient thermoreflectance from thin metal films

Journal of Applied Physics 60, 285 (1998); 10.1063/1.337642

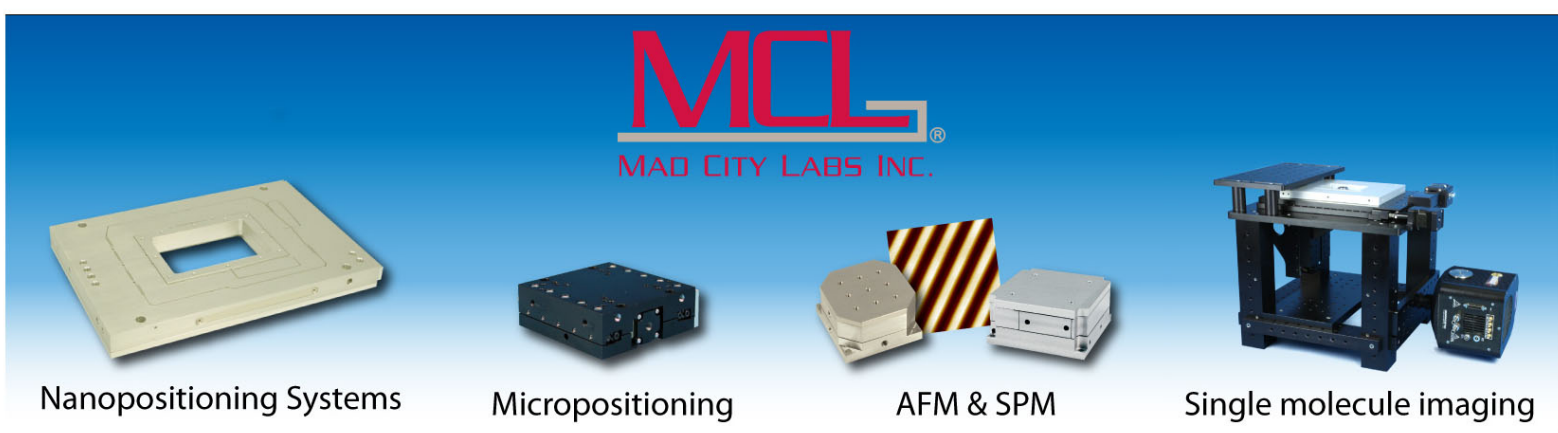




\title{
The study of frequency-scan photothermal reflectance technique for thermal diffusivity measurement
}

\author{
Zilong Hua, ${ }^{1}$ Heng Ban, ${ }^{1, a)}$ and David H. Hurley ${ }^{2}$ \\ ${ }^{1}$ Mechanical and Aerospace Engineering Department, Utah State University, Logan, Utah 84322-4130, USA \\ ${ }^{2}$ Materials Science and Engineering Department, Idaho National Laboratory, Idaho Falls, \\ Idaho 83415-2209, USA
}

(Received 7 January 2015; accepted 20 April 2015; published online 5 May 2015)

\begin{abstract}
A frequency scan photothermal reflectance technique to measure thermal diffusivity of bulk samples is studied in this manuscript. Similar to general photothermal reflectance methods, an intensitymodulated heating laser and a constant intensity probe laser are used to determine the surface temperature response under sinusoidal heating. The approach involves fixing the distance between the heating and probe laser spots, recording the phase lag of reflected probe laser intensity with respect to the heating laser frequency modulation, and extracting thermal diffusivity using the phase lag-(frequency) ${ }^{1 / 2}$ relation. The experimental validation is performed on three samples $\left(\mathrm{SiO}_{2}, \mathrm{CaF}_{2}\right.$, and $\mathrm{Ge}$ ), which have a wide range of thermal diffusivities. The measured thermal diffusivity values agree closely with the literature values. Compared to the commonly used spatial scan method, the experimental setup and operation of the frequency scan method are simplified, and the uncertainty level is equal to or smaller than that of the spatial scan method. (C) 2015 AIP Publishing LLC. [http://dx.doi.org/10.1063/1.4919609]
\end{abstract}

\section{INTRODUCTION}

The photothermal reflectance technique has become a promising tool for measuring thermal transport properties of bulk and thin film materials down to the microscale. The principle of the photothermal reflectance technique involves heating the sample with an intensity-modulated laser and using another constant intensity laser to detect the thermal wave propagation by optical reflectance. ${ }^{1,2}$ The relationship can be used to extract thermal diffusivity $(D)$ of bulk materials in a straight forward manner. ${ }^{3,4}$

If the sample has a layered structure, e.g., film-substrate, thermal diffusivities of different layers can also be determined through a depth-profiling approach. As the thermal diffusion length of the thermal wave decreases with its frequency, the probing depth can be controlled by adjusting the modulation frequency of the heating laser. For example, the response of a sample with a film-substrate structure is dominated by the film at high frequencies and by the substrate at low frequencies. Thus by measuring at different frequencies, thermal diffusivities of both layers can be obtained. The theoretical models with multi-layered structures have been developed by Reichling and Gronbeck, ${ }^{5}$ Lepoutre et al. ${ }^{6} \mathrm{Li}$ and Zhang ${ }^{7}$ and Xing et al. ${ }^{8}$ In those works, the influences of important experimental factors and various measurement strategies were discussed. Based on these theoretical models, a number of lab-oriented experiments were developed to extract thermal properties, ${ }^{9-13}$ measure interface thermal resistance, ${ }^{14-16}$ and detect invisible defects underneath the sample surface. ${ }^{17}$

The photothermal reflectance technique is known for its advantages such as non-contact, non-destructive nature and micrometer spatial resolution. However, the requirement of

a)Email: heng.ban@usu.edu controlling and metering the distance between laser spots at micrometer scale precisely, which is typically achieved by operating a motorized stage to move one of the laser beams before they enter the objective lens, has limited the measurement technique to be used only in the lab-oriented researches. No report has been published on its use in industry or in situ applications so far, partly because the use of the motorized stage complicates the experimental design and operation and may introduce measurement uncertainty.

The frequency scan photothermal reflectance method has the potential to overcome the shortcomings of the spatial scan method. With a fixed distance between laser spots and varying the modulation frequency of the heating laser, thermal diffusivity can be extracted from the phase lag-(frequency $)^{1 / 2}$ relation. This method was first, and only, used to study the thermal diffusivity of superconductor, ${ }^{18}$ while the measurement technique approach has not been discussed before. In this paper, the frequency scan photothermal reflectance method is discussed in detail. The approach guideline is given and validated on $\mathrm{SiO}_{2}, \mathrm{CaF}_{2}$, and $\mathrm{Ge}$. In order to improve the optical absorption, $\mathrm{SiO}_{2}$ and $\mathrm{CaF}_{2}$ samples were coated by $100 \mathrm{~nm}$ thick titanium films, from which the capability of this method to measure substrate thermal diffusivity on layered samples was also examined. As the motorized stage is no longer necessary, the experimental design and operation are simplified, and the system can be more compact, which provides potentials for wider applications.

\section{THEORY}

Theoretical models of photothermal reflectance techniques have been developed in the past studies. The theoretical basis of the frequency scan method is a straightforward variation of existing models. As in Figure 1, assuming a 


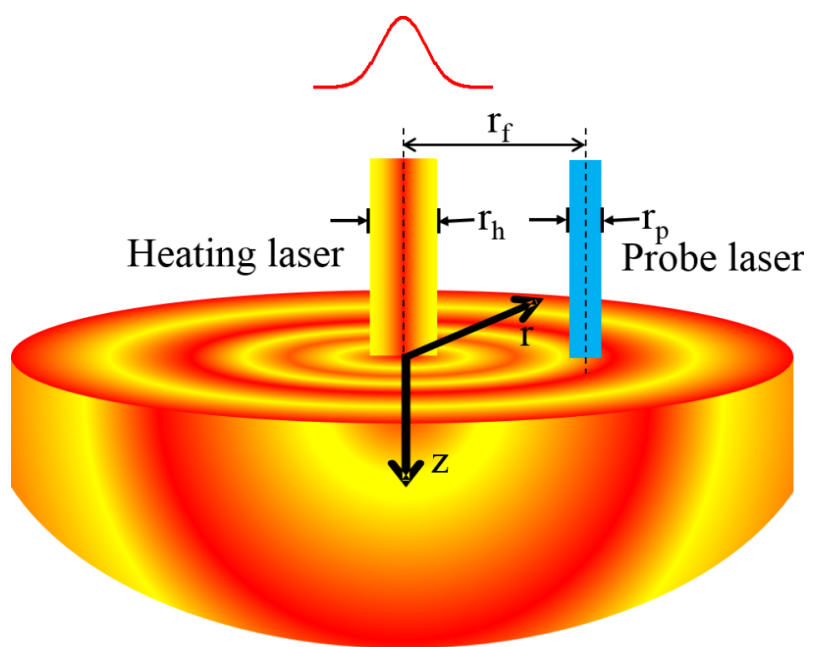

FIG. 1. Description of the measurement principle: a spatially Gaussian distributed heat source (Gaussian radius $r_{h}$ ) is applied on the sample surface; the intensity of the heat source is modulated periodically; the probe with a Gaussian radius $r_{p}$ is located $r_{f}$ away from the heat source.

homogenous and isotropic material is heated on the surface by a harmonic heat source with Gaussian intensity distribution (radius $r_{h}$ ), the periodic component of the temperature response in a detection area with a Gaussian radius of $r_{p}$ can be described by the heat conduction equation in cylindrical coordinate as

$$
\frac{1}{r} \frac{\partial}{\partial r}\left(r \frac{\partial T}{\partial r}\right)+\frac{\partial^{2} T}{\partial z^{2}}-\frac{i \omega}{D} T=0 .
$$

With boundary conditions $\left.T(r, z)\right|_{z \rightarrow \infty}=0$ and $-\left.k \frac{\partial T(r, z)}{\partial z}\right|_{z=0}$ $=P_{0} \exp \left(-\frac{2 r^{2}}{r_{0}^{2}}\right)$, where $\omega$ and $P_{0}$ are the angular frequency and power of the heat source, respectively, and $r_{0}$ is the effective radius $\left(r_{0}=\sqrt{\left(r_{h}^{2}+r_{p}^{2}\right)}\right)$, the solution can be derived through Hankel transformation as ${ }^{6}$

$$
\begin{aligned}
T(r, z)= & \frac{P_{0} r_{0}^{2}}{4 k} \int_{0}^{\infty} \frac{1}{\sqrt{s^{2}+\frac{i \omega}{D}}} \exp \left(-\frac{\mathrm{s}^{2} \mathrm{r}_{0}^{2}}{8}\right) \\
& \times \exp \left(-z \sqrt{s^{2}+\frac{i \omega}{D}}\right) \mathrm{J}_{0}(\mathrm{rs}) \mathrm{sds},
\end{aligned}
$$

where $s$ is the Hankel transformation variable. Although Eq. (2) can only be solved numerically, the thermal wave phase lag-detection location $(\varphi-r)$ relation is available by setting $z=0$ and performing inverse Hankel transform on the $\frac{1}{\sqrt{\mathrm{s}^{2}+\frac{\mathrm{i}}{\mathrm{D}}}}$ terms as

$$
\frac{d \varphi}{d r}=-\sqrt{\frac{\omega}{2 D}}=\sqrt{\frac{\pi f}{D}} .
$$

Therefore, by recording the phase lags corresponding to the distance of the heating and detection spots, thermal diffusivity can be extracted from $d \varphi / d r$ in Eq. (3) or the slope of the $\varphi-r$ curve as in Figure 2. In the typical photothermal reflectance technique, it is achieved by spatially scanning one of the laser beams across the other one. Note the slope is also a function of the frequency of the heat source $(f)$. In Figure 2, the phase lags collected at three frequencies show

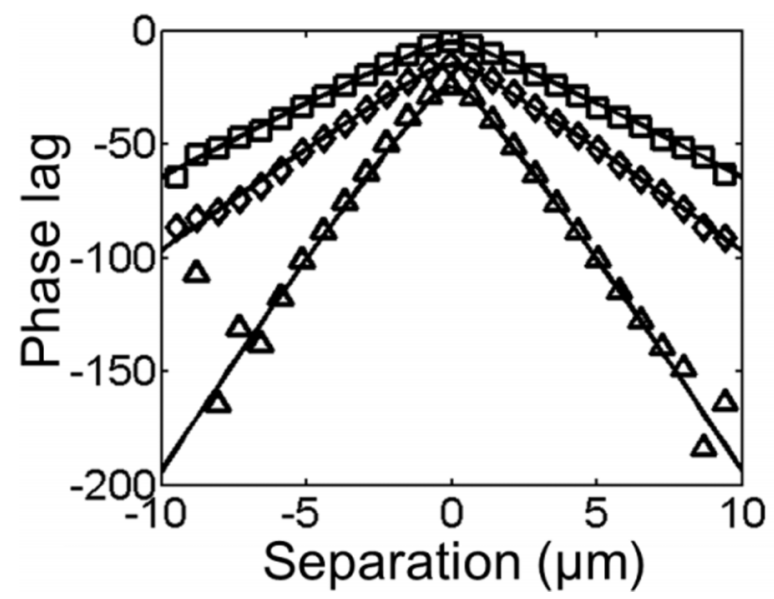

FIG. 2. An example of phase lag-laser separation curves from spatial scan photothermal reflectance measurements on $\mathrm{SiO}_{2}$. The measurements are taken at three frequencies $(5 \mathrm{kHz}, 10 \mathrm{kHz}$, and $50 \mathrm{kHz})$.

different slopes. If $f$ in Eq. (3) is considered as the variable, Eq. (3) can be rewritten as Eq. (4), suggesting to use the phase lag-(frequency $)^{1 / 2}$ relation $\left(\varphi-f^{1 / 2}\right)$ for the thermal diffusivity extraction, if the separation between the heat source and detection spot is fixed as $r_{f}$ (as in Figure 1),

$$
\frac{d \varphi}{d \sqrt{f}}=-r_{f} \sqrt{\frac{\pi}{D}}
$$

Comparing to the spatial scan method, which requires to adjust the frequency of the heating laser intensity (the measurements are always performed at different frequencies to improve the measurement accuracy) and position of one of the laser beams, in the frequency scan method, once heating and probe beams are separated by a distance of $r_{f}$, it is only the frequency of the heating laser intensity that needs to be changed; thus, the experiment procedure is simplified. The separation distance $r_{f}$ is found the most important parameter in frequency scan measurements for reasons below. First, it decisively influences the measurement sensitivity and the signal-to-noise ratio (SNR) that the measurement accuracy mainly depends on. It is known that the amplitude of the thermal wave attenuates with propagation distance, while sensitivities of interested thermal properties are proportional to the distance between laser spots. ${ }^{19}$ In addition, a proper $r_{f}$ can greatly decrease the measurement error of using Eq. (4) in the fitting process. Eq. (2) can precisely describe the heat conduction in actual measurements (due to the use of laser beams which have Gaussian spatial distribution) but it needs to numerically integrate to solve. Eq. (4), a simplified form of Eq. (2) by considering the heat source and probe as points without size or distribution, produces less than $1 \%$ error if $r_{f}$ is properly set. As the $\varphi-f$ relationship exists explicitly in Eq. (4) but not in Eq. (2), it is more efficient and convenient to use Eq. (4) in the fitting process. More details of the $r_{f}$ determination will be discussed later in Sec. III.

\section{EXPERIMENTAL SETUP}

The experimental setup is similar as the one in Ref. 19. The output powers of the heating and probe lasers on the 


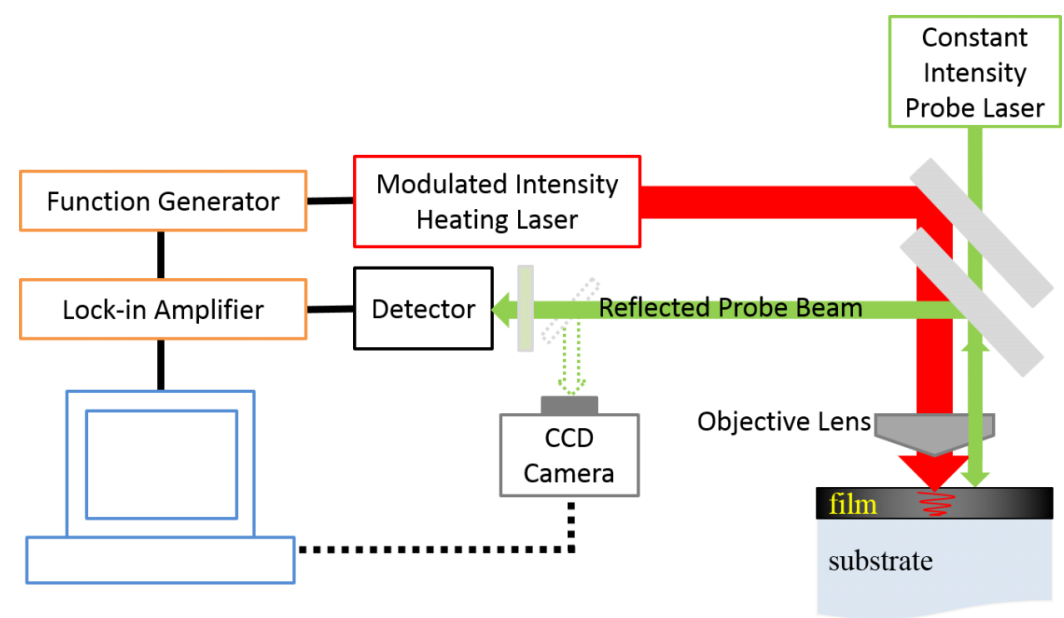

FIG. 3. Design diagram of the experimental setup.

sample surface are $\sim 2 \mathrm{~mW}$ and $\sim 1 \mathrm{~mW}$, respectively, measured by a power meter. In order to improve the spatial resolution, both laser beams are focused by a $50 \times$ objective lens, and the radius of each laser spot on the surface is about $1 \mu \mathrm{m}$. A mechanical XYZ-stage is used to adjust the distance from the probe laser beam to the heating one in order to test the effect of different separation distances in this experiment. In the actual measurement, this distance is fixed; thus, the stage is not necessary, and the size and complexity of the measurement setup are reduced from the typical spatial scan method.

The design diagram of the setup is given in Figure 3. The intensity of the heating laser is modulated by an acoustooptic modulator (AOM), and its amplitude and frequency are controlled by the waveform generator. The reflected probe laser beam from the sample surface is collected by a detector and a lock-in amplifier to analyze the phase $\left(\varphi_{s}\right)$ after passing a bandpass filter to screen the reflected heating laser beam. The system dependent phase shift (instrument factor, $\varphi_{i f}$ ) is obtained by turning off the probe laser beam and removing the filter thus measuring the phase of the reflected heating laser beam directly. Note the light paths of the heating and probe laser beams are adjusted equal to each other. The difference of the signal phase to the system phase shift $\left(\varphi_{s}-\varphi_{i f}\right)$ is the phase lag discussed in the theoretical model previously, which is used to extract the thermal diffusivity.

As stated previously, the key to measurement accuracy is the laser spot separation distance $r_{f}$ that is determined from a SNR test. SNR used in this manuscript is defined as the ratio of intensities (which is read by the lock-in amplifier with a time constant of $3 \mathrm{~s}$ ) of the reflected probe laser beam with to without the heat source. Based on this definition, the noise level is found primarily dependent on the noise spectrum of the probe laser, while the heating laser performance, the sample surface quality, and the distance between heating and detection spots $\left(r_{f}\right)$ are minor contributors to the measurement noise. A noise test of the probe laser suggested a lower frequency limit to be $100 \mathrm{~Hz}$, as the noise is consistent at a low level above $100 \mathrm{~Hz}$ but rapidly increases when the frequency drops below $100 \mathrm{~Hz}$ (Figure 4). The critical factors of the signal level are the thermal wave amplitude and response time of the instruments, both of which decrease with frequency. After repeated tests, the upper limit of the measurement frequency range was determined to be $10 \mathrm{kHz}$.

The SNR test is performed in the frequency range of $100 \mathrm{~Hz}-10 \mathrm{kHz}$, and $\mathrm{CaF}_{2}$ is selected as the reference test sample. As a rule of thumb, SNR above 10 is considered sufficient for photothermal reflectance measurements. Since the thermal wave amplitude and sensitivity of the measurement are expected to change with $r_{f}$ in opposite directions, the essential criterion of the $r_{f}$ determination is to find the balance point between the two from the test. With the consideration of keeping SNR above 10 in the entire frequency range, $r_{f}$ is chosen to be $\sim 20 \mu \mathrm{m}$. It is worth noting that the measurement frequency range and $r_{f}$ are slightly influenced by the setup instruments and the test sample. In different experimental environments, SNR test results are expected to be different.

In principle, the Gaussian spatial distribution of heating and probe laser beams' intensity makes Eq. (2) a more precise description of the heat conduction problem in this manuscript. However, if the laser beam separation $r_{f}$ is large enough comparing to the effective beam size $r_{0}$, the heat source and probe can be considered as points and the difference of using either equation (Eq. (2) or Eq. (4)) is negligible. The

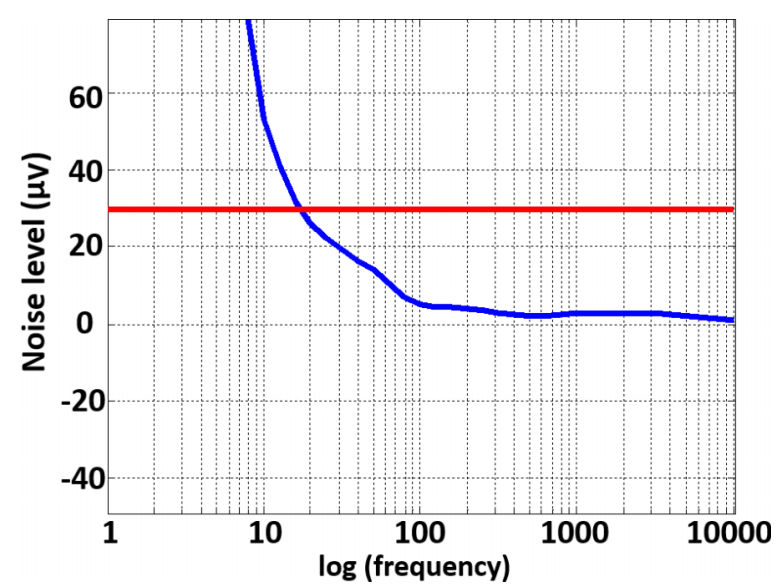

FIG. 4. The noise level of the measurement setup. A horizontal red line is added as reference which represents the necessary signal level to satisfy the critical SNR. It can be seen that the noise increases with decreasing frequency dramatically below $100 \mathrm{~Hz}$. 


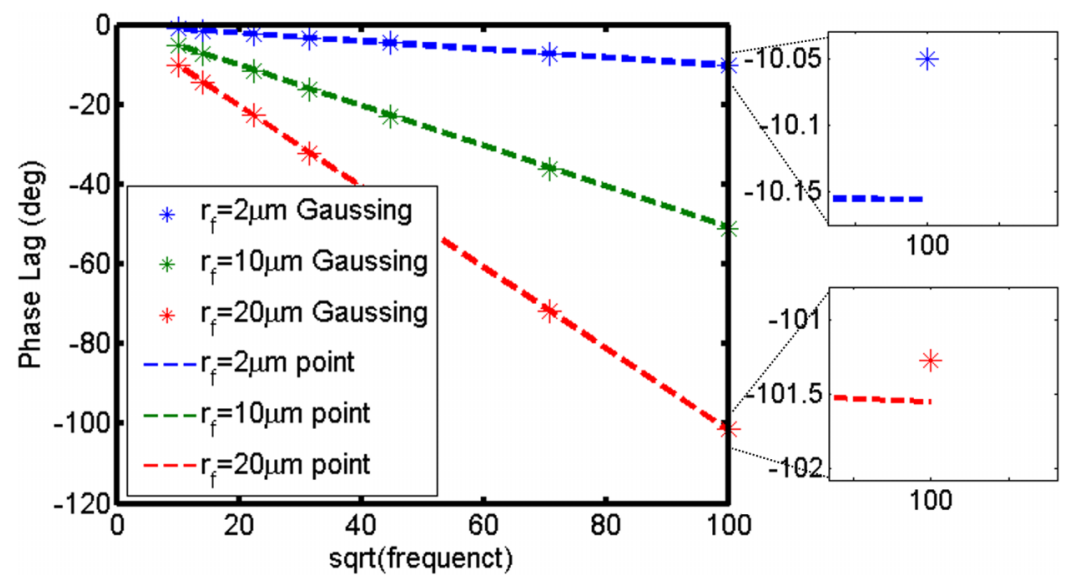

FIG. 5. Simulation results from different theoretical models with different laser separations (dashed line-point heating/probe laser which is described by Eq. (4); star—spatially Gaussian distributed heating/probe laser). With the heating laser radius of $1 \mu \mathrm{m}$, the phase-lag difference (percentage) decreases with increasing laser separation from $1 \%$ when $r_{f}=2 \mu \mathrm{m}$ to $0.3 \%$ when $r_{f}=20 \mu \mathrm{m}$.

simulation results on $\mathrm{CaF}_{2}$ sample are plotted in Figure 5. The radii of both laser beams in the simulation are set $1 \mu \mathrm{m}$, similar as the ones in the real experiment. With the laser separation $\left(r_{f}\right)$ increasing from $2 \mu \mathrm{m}$ to $10 \mu \mathrm{m}$ and then $20 \mu \mathrm{m}$, the difference between two models is found decreasing from $1 \%$ at $r_{f}=2 \mu \mathrm{m}$ to $0.3 \%$ at $r_{f}=20 \mu \mathrm{m}$. At the upper limit of $10 \mathrm{kHz}$, the absolute difference $\left(\sim 0.3^{\circ}\right)$ is smaller than the measurement uncertainty from the instruments $\left(\sim 0.5^{\circ}\right)$. Therefore, with the experimental condition in this manuscript, it is safe to use Eq. (4) instead of Eq. (2) to extract the thermal diffusivity without introducing noticeable error.

\section{RESULTS AND DISCUSSION}

$\mathrm{SiO}_{2}, \mathrm{CaF}_{2}$, and $\mathrm{Ge}$, with thermal diffusivities of 9.5 $\times 10^{-7} \mathrm{~m}^{2} / \mathrm{s}, 3.7 \times 10^{-6} \mathrm{~m}^{2} / \mathrm{s}$, and $3.6 \times 10^{-5} \mathrm{~m}^{2} / \mathrm{s}$, respectively, ${ }^{19,20}$ are used to validate the measurement approach. In order to improve the laser absorption and thermoreflectance effect, a thin film layer of titanium was added on $\mathrm{SiO}_{2}$ and $\mathrm{CaF}_{2}$ samples with a thickness of $100 \mathrm{~nm}$. Titanium was selected as the coating material for its good thermoreflectance coefficient of $-5 \times 10^{-5} \mathrm{~K}^{-1}$ at the probe laser wavelength $(660 \mathrm{~nm}){ }^{21}$ A set of measurements are performed on each sample at 11 different frequencies from $25 \mathrm{~Hz}$ to $10 \mathrm{kHz}$. The data below $100 \mathrm{~Hz}$ are collected to illustrate the situation with a low SNR (the SNR at $25 \mathrm{~Hz}$ is $\sim 1$ ). Comparing to the spatial scan method in which the phase lags are measured at several tens of spots at each frequency, in the frequency scan method, phase lags at only one location need to be recorded. Although a longer time constant of the lock-in amplifier is used to control the noise level, the time consumption to complete the measurement is still significantly reduced from 30 to $60 \mathrm{~min}$ to less than 10 min.

As in Figure 6, the thermal diffusivities of validation samples are extracted from a least-square fitting process in which the experimental data are compared to the simulation results from Eq. (4). The best-fit results are summarized with the literature values in Table I. The differences between the fitting results and the literature values are no more than $3 \%$, generally better than the spatial scan method. The smallest difference is found on the $\mathrm{SiO}_{2}$ sample $(0.6 \%)$, suggesting a better measurement accuracy on low thermal diffusivity samples. It is because the slope of phase lag-(frequency) $)^{1 / 2}$ curve is inversely proportional to (thermal diffusivity) $)^{1 / 2}$. With the similar noise level, the steep slope on materials associated with low thermal diffusivity reduces the weight of noise in the measured phase lags. Although the spatial resolutions of both methods are in the micrometer level, thus the measured values reflect the local thermal diffusivity that varies with locations, it is still reasonable to conclude that the frequency scan measurement has at least the same or better precision than that of the typical spatial scan measurement. In addition, the smaller (average) distance between heating and probe laser spots in the spatial scan measurements can cause a higher background temperature rise, which will theoretically decrease the measurement accuracy if thermal properties of the sample are sensitive to temperature. For instance, as discussed in Ref. 19, this factor was believed to be one of the reasons why the measurement accuracy of the $\mathrm{CaF}_{2}$ sample is lower than that of the $\mathrm{SiO}_{2}$ sample.

The fitting curves in Figure 6 are close to most data spots except for some obtained below $400 \mathrm{~Hz}$, which proves the importance of a sufficient SNR to the experimental results.

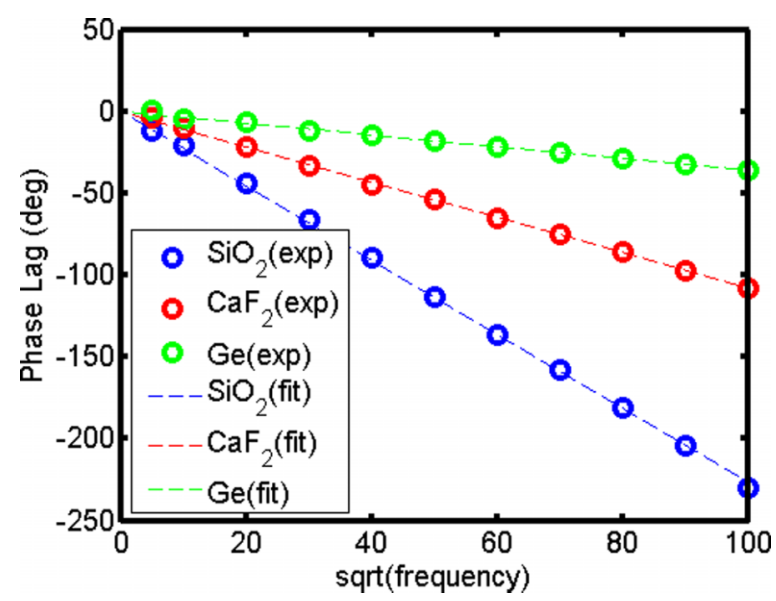

FIG. 6. Experimental results of $\mathrm{SiO}_{2}, \mathrm{CaF}_{2}$, and $\mathrm{Ge}$ samples with leastsquare fitting curves (circle—experimental data; dashed line—-fitting curves). 
TABLE I. Experimental results.

\begin{tabular}{lcccc}
\hline \hline Sample & Measured D & $\begin{array}{c}\text { Literature } \\
\text { D }\end{array}$ & $\begin{array}{c}\text { Difference } \\
(\%)\end{array}$ & $\begin{array}{c}\text { Difference (Spatial } \\
\text { Scan method) }\end{array}$ \\
\hline $\mathrm{SiO}_{2}$ & $9.44 \times 10^{-7}$ & $9.5 \times 10^{-7}$ & $\sim 0.6$ & $3 \%{ }^{19}$ \\
$\mathrm{CaF}_{2}$ & $3.58 \times 10^{-6}$ & $3.7 \times 10^{-6}$ & $\sim 3$ & $4 \% 19$ \\
$\mathrm{Ge}$ & $3.70 \times 10^{-5}$ & $3.6 \times 10^{-5}$ & $<2.8$ & N/A \\
\hline \hline
\end{tabular}

Besides a low SNR, another possible reason of the relatively large fluctuation at a low frequency is the breakdown of the semi-infinite assumption used in the analytical model. Thermal diffusion lengths of $\mathrm{CaF}_{2}$ and $\mathrm{Ge}$ are $230 \mu \mathrm{m}$ and $700 \mu \mathrm{m}$, respectively, at $25 \mathrm{~Hz}$, comparable to the sample thicknesses of $1 \mathrm{~mm}$, which makes the thermal wave more likely to penetrate the whole sample and reach the back side, causing the failure of the semi-infinite boundary condition applied in the derivation. As a comparison, the thermal diffusion lengths are $110 \mu \mathrm{m}$ for $\mathrm{SiO}_{2}$ at $25 \mathrm{~Hz}$ and $\mathrm{Ge}$ at $900 \mathrm{~Hz}$, where the fitting results match the experimental data with negligible difference. Therefore, using the best-fit thermal diffusivity to check the thermal diffusion length at the lower frequency limit is very important after the fitting process. If the thermal diffusion length is found to be comparable to the geometry size of the sample, it is necessary to perform the fitting process again with a higher starting frequency. Similar to the minimum SNR value of 10 , a ratio of 10 between the sample thickness and the largest thermal diffusion length (at the lower frequency limit) is necessary to ensure the validity of the theory. The importance of these critical ratios is illustrated in the offset analysis. A pair of negative and positive curves which are fitted from the single data spots with the maximum deviation comparing to the best-fit curve are plotted in Figure 7, and the results are summarized in Table II. Although the data from below $900 \mathrm{~Hz}$ are not included, larger offsets are still found on the spots at or near both ends of the frequency region where either SNR or the ratio of the sample thickness to the thermal diffusion length is low.

The measurement uncertainty of thermal diffusivity $D$ can be calculated from Eq. (4) using the individual uncertainties of the phase lags, $\varphi$, the laser spot separation distance, $r_{f}$, and the measurement frequency, $f$. In our measurement, $f$ is controlled by the waveform generator and has a negligible uncertainty of less than $0.1 \mathrm{~Hz} ; r_{f}$ is adjusted by a mechanical XYZ-stage whose uncertainty is less than $0.5 \mu \mathrm{m}$; after performing the measurements on $\mathrm{CaF}_{2}$ sample at 10 different locations, the average value (at 11 data spots) of the phase lag standard error of the mean is found to be less than $2.5 \%$. Using these values, the measurement uncertainty of $D$ is calculated to be $\sim 2.7 \%$, dominated by the phase lag uncertainty. The uncertainty of each parameter is strongly dependent on the instruments and can be optimized. For instance, if a longer time constant on the lock-in amplifier is used to decrease the phase lag standard error of the mean to $\sim 1 \%$, the overall measurement uncertainty will go down to $\sim 1.5 \%$.

One possible source of the experimental error that has little influence on our measurement but may cause remarkable error in other setups is the size of the laser spots. In the spatial scan photothermal reflectance measurement, it is found that the experimental data obtained from or close to the area where the heating and probe laser spots overlap each
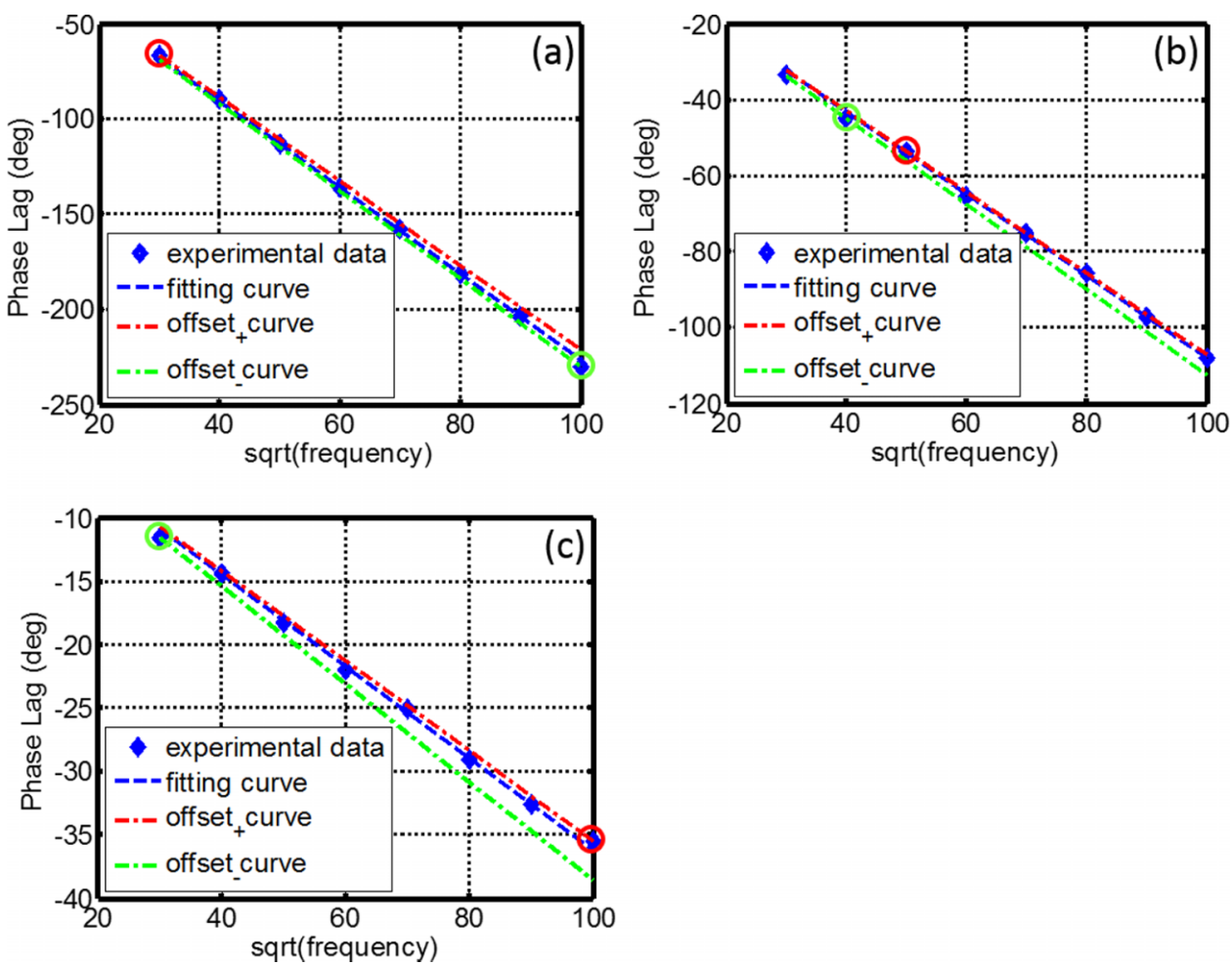

FIG. 7. Plots with offset curves of three samples: (a) $\mathrm{SiO}_{2}$, (b) $\mathrm{CaF}_{2}$, and (c) Ge. Two offset curves are plotted with the experimental data and fitting curves (diamond—experimental data; dashed line—fitting curves; dotted-dashed line—offset curves). 
TABLE II. Summary of uncertainty analysis (decided by offset curves).

\begin{tabular}{lcccccc}
\hline \hline Sample & Offset+ & Offset $\%$ & Freq $_{\text {off }}{ }^{+}$ & Offset- & Offset $\%^{\text {Freq }_{\text {off }}}$ \\
\hline $\mathrm{SiO}_{2}$ & $9.90 \times 10^{-7}$ & $\sim 4.9$ & $900 \mathrm{~Hz}$ & $9.15 \times 10^{-7}$ & $\sim 3.1$ & $10 \mathrm{kHz}$ \\
$\mathrm{CaF}_{2}$ & $3.56 \times 10^{-6}$ & $\sim 1.2$ & $2.5 \mathrm{kHz}$ & $3.24 \times 10^{-6}$ & $\sim 7.9$ & $1.6 \mathrm{kHz}$ \\
$\mathrm{Ge}$ & $3.85 \times 10^{-5}$ & $\sim 4.1$ & $10 \mathrm{kHz}$ & $3.26 \times 10^{-5}$ & $\sim 11.9$ & $900 \mathrm{~Hz}$ \\
\hline \hline
\end{tabular}

other contain seldom useful information for the thermal properties extraction. A similar effect exists in the frequency scan measurements. The difference caused by the spot size alone is simulated on the $\mathrm{CaF}_{2}$ sample and the results are shown in Figure 8. In the simulation, the heating laser spot size $r_{h}$ is changed from $1 \mu \mathrm{m}$ to $2 \mu \mathrm{m}$ and then $5 \mu \mathrm{m}$, respectively, while the probe laser spot size is kept as $1 \mu \mathrm{m}$, and $r_{f}$ is set as $10 \mu \mathrm{m}$. At $10 \mathrm{kHz}$, the phase lag difference between two models (with point heat source/probe and Gaussian heat source/probe) is $\sim 1.5^{\circ}$, or $3 \%$ if $r_{h}$ is $2 \mu \mathrm{m}$, and $\sim 6.5^{\circ}$, or $13 \%$ if $r_{h}$ is $5 \mu \mathrm{m}$. It is large enough to cause a noticeable "bending" on the curve which will influence the fitting process and introduce measurement error. In order to decrease this type of error, it is recommended to use heating and probe lasers with small beam radii. Otherwise, a larger ratio between $r_{f}$ and the laser beam spot sizes is necessary. Keep the heating laser spot size at $5 \mu \mathrm{m}$, the phase lag difference decreases from $13 \%$ to $4 \%$ by doubling $r_{f}$.

Although properties of the coating film are known to influence the measurement results in the high frequency region, ${ }^{19}$ no noticeable deviation is found on $\mathrm{SiO}_{2}$ and $\mathrm{CaF}_{2}$ samples between their fitting curves and experimental results at the upper limit of $10 \mathrm{kHz}$. The primary reason is that the Ti film has relatively low thermal diffusivity, which in addition to its 100-nm thickness makes the film thermally thin. Our simulation shows that a 100 -nm thick Ti layer only causes a $8.5 \%$ difference of the phase lag measured on $\mathrm{SiO}_{2}$ at $10 \mathrm{kHz}$ (approximately from $-200^{\circ}$ to $-183^{\circ}$ ). Sensitivity analysis on the $\mathrm{Ti}_{-} \mathrm{SiO}_{2}$ sample leads to the same conclusion. The sensitivity of the substrate thermal diffusivity dominates over the entire frequency region. Consequently, if the sample is coated

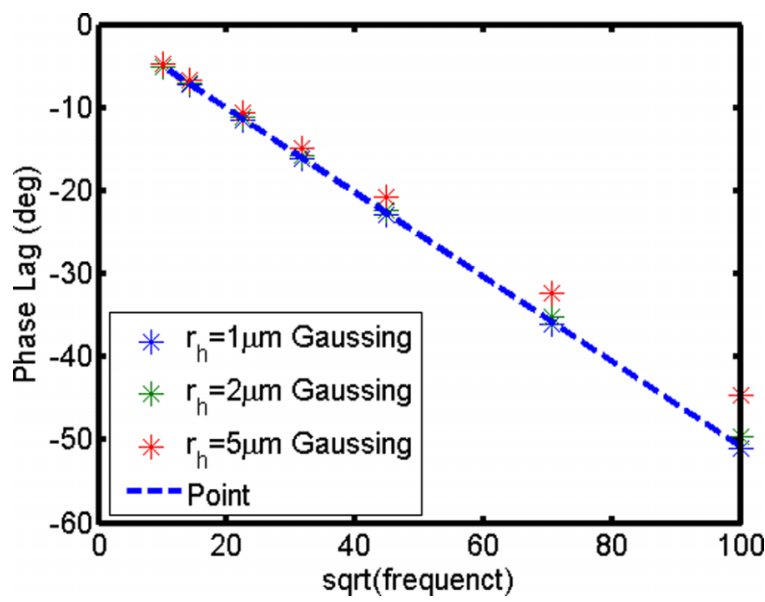

FIG. 8. The influence of laser spot sizes on measurement results (starGaussian distributed heat source and probe; dashed line-point heat source and probe). It can be seen that the difference of the simulation results between different models increases with the radius of the heat source area. by a thermally thin film (such as the 100-nm Ti film in our study), the frequency scan method is always more sensitive to substrate properties than film ones, which makes it a candidate to measure substrate thermal diffusivity. In case, a material with high thermal conductivity and diffusivity is the only available option for coating, in order to guarantee the measurement accuracy, the film needs to be made very thin.

\section{CONCLUSION}

In this paper, the frequency scan photothermal reflectance technique based thermal diffusivity measurement approach is studied in detail and validated. Comparing to the commonly used spatial scan technique, the system setup and measurement procedure are simplified. Moreover, the accuracy of the experimental result is better, especially for the samples of which thermal properties have strong temperature dependence. Following the proper guideline, the substrate thermal diffusivity of coated samples can be obtained with good accuracy. This frequency scan method has important technological implications for advanced material properties evaluation at the microscale.

${ }^{1}$ A. Rosencwaig, J. Opsal, W. L. Smith, and D. Willenborg, Appl. Phys. Lett. 46(11), 1013 (1985).

${ }^{2}$ C. A. Paddock and G. L. Eesley, J. Appl. Phys. 60(1), 285 (1986).

${ }^{3} \mathrm{H}$. Carslaw and J. Jaeger, Conduction of Heat in Solids (Oxford University Press, London, 1959).

${ }^{4}$ A. Salazar, Eur. J. Phys. 27(6), 1349 (2006).

${ }^{5}$ M. Reichling and H. Gronbeck, J. Appl. Phys. 75(4), 1914 (1994).

${ }^{6}$ F. Lepoutre, D. Balageas, P. Forge, S. Hirschi, J. Joulaud, D. Rochais, and F. Chen, J. Appl. Phys. 78(4), 2208 (1995).

${ }^{7}$ B.-c. Li and S.-y. Zhang, J. Phys. D: Appl. Phys. 30(10), 1447 (1997).

${ }^{8}$ C. Xing, C. Jensen, Z. Hua, H. Ban, D. Hurley, M. Khafizov, and J. R. Kennedy, J. Appl. Phys. 112, 103105 (2012).

${ }^{9}$ P. Alpern and S. Wurm, J. Appl. Phys. 66(4), 1676 (1989).

${ }^{10}$ A. Maznev, J. Hartmann, and M. Reichling, J. Appl. Phys. 78(9), 5266 (1985).

${ }^{11}$ T. Yagi, N. Taketoshi, and H. Kato, Physica C 412, 1337 (2004).

${ }^{12}$ K. Hatori, N. Taketoshi, T. Baba, and H. Ohta, Rev. Sci. Instrum. 76(11), 114901 (2005).

${ }^{13}$ A. Yarai and T. Nakanishi, Rev. Sci. Instrum. 78(5), 054903 (2007).

${ }^{14}$ A. Mansanares, T. Velinov, Z. Bozoki, D. Fournier, and A. Boccara, J. Appl. Phys. 75(7), 3344 (1994)

${ }^{15}$ B. Li, J. Roger, L. Pottier, and D. Fournier, J. Appl. Phys. 86(9), 5314 (1999).

${ }^{16}$ D. Hurley, M. Khafizov, and S. Shinde, J. Appl. Phys. 109, 083504 (2011).

${ }^{17}$ J. Bailey, E. R. Weber, and J. Opsal, J. Cryst. Growth 103(1), 217 (1990).

${ }^{18}$ J. Fanton, D. Mitzi, A. Kapitulnik, B. Khuri-Yakub, G. Kino, D. Gazit, and R. Feigelson, Appl. Phys. Lett. 55(6), 598 (1989).

${ }^{19}$ Z. Hua, H. Ban, M. Khafizov, R. Schley, R. Kennedy, and D. H. Hurley, J. Appl. Phys. 111(10), 103505 (2012).

${ }^{20}$ Y. S. Touloukian, R. W. Powell, C. Y. Ho, and M. C. Nicolaou, Thermophysical Properties of Matter-The TPRC Data Series. Volume 10. Thermal Diffusivity, DTIC Document (1973).

${ }^{21}$ M. G. Burzo, P. L. Komarov, and P. E. Raad, in IEEE Twenty-Second Annual, Semiconductor Thermal Measurement and Management Symposium (IEEE, 2006), p. 87. 